\title{
Compact Planar Integrated Rectenna for Batteryless IoT Applications
}

\author{
A. Takacs", A. Okba ${ }^{\#}$, H. Aubert

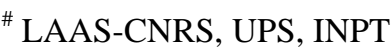 \\ Toulouse, France \\ aokba@laas.fr, atakacs@laas.fr, aubert@laas.fr
}

\begin{abstract}
This paper addresses a new topology of compact rectennas in which the rectifier is integrated directly on the radiating surface. The rectenna is designed for wireless power transmission or microwave energy harvesting application in ISM $900 \mathrm{MHz}$ band and exhibits a very good measured conversion efficiency $(>25 \%)$ on a non-optimal load $(10 \mathrm{k} \Omega)$ for very low microwave power densities $\left(>0.18 \mu \mathrm{W} / \mathrm{cm}^{2}\right)$. The measured DC voltage $(>330 \mathrm{mV}$ for microwave power density of at least 0.22 $\mu W / \mathrm{cm}^{2}$ ) obtained from this planar rectenna is in the range of the cold turn-on/start-up voltage of modern commercial off-the-shelf DC-to-DC boost converters and power management units. The proposed rectenna is also very compact: its physical surface (10.5 x $\left.6 \mathrm{~cm}^{2}\right)$ is only $5 \%$ of the square wavelength at the operating frequency $(860 \mathrm{MHz})$.
\end{abstract}

Keywords - wireless power transmission, microwave energy harvesting, rectenna, internet of things.

\section{INTRODUCTION}

The rise of the Internet of Things (IoT) applications faces to a new challenge: how to power efficiently an enormous number of wireless sensors, intelligent tags, and devices? Nowadays, the use of a battery is almost a standard but, the topic of the self-powered/batteryless devices excites from longtime the scientific community and becomes recently an industrial reality for emerging innovative start-up and companies [1][2]. One solution to implement self-powered and/or batteryless devices is to use a wireless power transmission approach: an intentionally microwave transmitter (energy shower) will energize at distance a rectenna module (rectifier + antenna). The energy shower should operate in the ISM bands and is then subject to regulations. ISM 868/915 $\mathrm{MHz}$ band provides a good trade-off in terms of free-space losses (fixing the maximum operating range of such a system) and wavelength (fixing the size of receiving antenna of the rectenna module). Many rectenna designs operating in the ISM 868/915 MHz band were proposed in the past with a focus mainly on the measured intrinsic performances (e.g., efficiency, harvested DC power, etc.) [3]-[5]. From an industrial point of view, a successful rectenna design for IoT applications should be compact, low-profile and low-cost. The size of the rectenna module is determined mainly by the receiving antenna. Electronic devices should be also integrated with the antenna, e.g., the rectifier including its matching circuit, the Power Management Unit (PMU), the energy storage element, etc. But with the same goal in mind: keeping the overall structure as compact as possible.

This paper addresses the design in the ISM 868/915 MHz band of a compact rectenna using a miniaturized flat dipole antenna with the rectifier circuit directly integrated on the radiating surface of the antenna. To the authors' best knowledge, there are very few designs integrating the rectifier into radiating element at such low frequencies. In [6] the rectifier and the corresponding matching circuit are integrated in the ground plane of a patch antenna designed at $1.96 \mathrm{GHz}$ but they are not integrated in the patch surface itself. Very recently, V. Palazzi et al. [7] reported a compact rectenna using an annular slot with a wire-connected multibandrectifier positioned on the back-side of the antenna.

Our proposed innovative topology is described in Section II while the experimental results obtained with the manufactured prototype are presented and discussed in Section III.

\section{RECTENNA TOPOLOGY AND DESIGN}

A typical topology for an IoT compatible wireless sensor/tag is sketched in Fig.1. From a practical point of view, if a compact planar structure is desired, the main design objective is to integrate all the functions/electronics (e.g., rectifier, PMU, energy storage, wireless sensor/tag, etc.) into the same Printed Circuit Board (PCB). The size of the overall structure is then mainly fixed by the size of the antenna operating at lowest frequency.

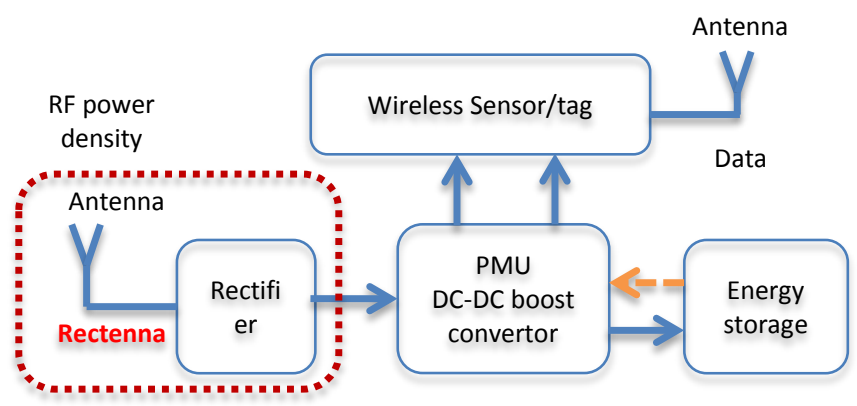

Fig. 1. Sketch of the typical topology of a wireless sensor/tag for an IoT scenario 
As design requirements, we target a compact and fully planar rectenna with optimized performances in the ISM 868/915 MHz band. This rectenna should be able to power a standard DC-to-DC boost convertor and PMU (such as, e.g., BQ25504 device [8]) for incident electromagnetic (EM) power densities around $1 \mu \mathrm{W} / \mathrm{cm}^{2}$. Thus, the rectenna should provide an output DC voltage greater that the cold start-up voltage of such PMU (approximately $330 \mathrm{mV}$ for the BQ25504 device) on a high impedance load (typically $>9 \mathrm{k} \Omega$, corresponding to the PMU input impedance) with the highest efficiency. The topology of the proposed 2D compact planar integrated rectenna is shown in Fig. 2 with the 3D rectenna topology.

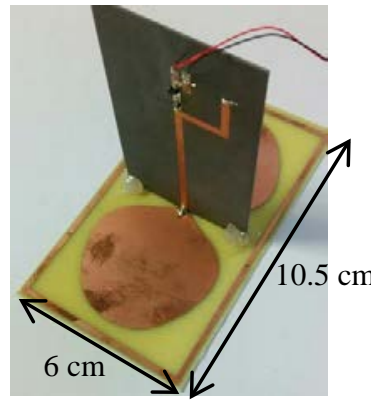

(a)

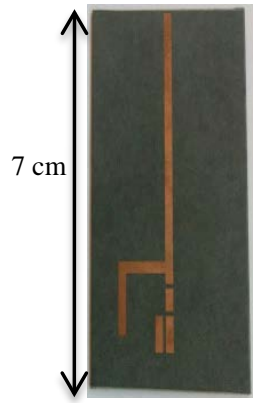

(b)

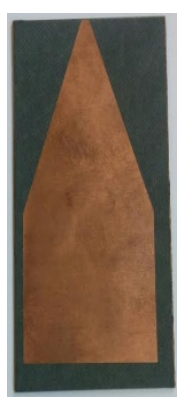

(c)

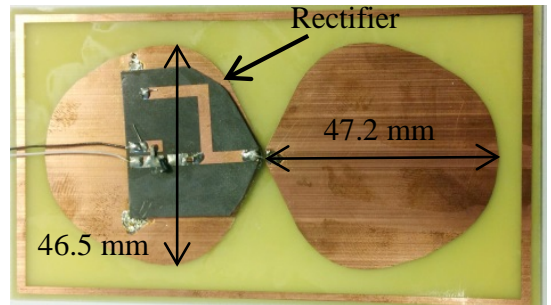

(d)

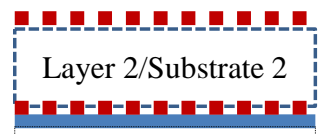

Layer $1 /$ Substrate 1
Fig. 2. The manufactured rectennas: (a) the $3 \mathrm{D}$ version of the rectenna combining a flat dipole antenna (surrounded by a rectangular ring) with a rectifier; (b) top view of the rectifier before the mounting of the lumped devices; (c) bottom view of the rectifier; (d) top view of the 2D rectenna and (e) cross sectional view of this rectenna (the continuous blue line corresponds to the metal layer while the dotted red lines indicate the metallic parts of the rectifier.

The rectenna consists of a flat dipole antenna (surrounded by a rectangular ring) integrated with a rectifier using a Schottky diode. The metal layer of the antenna is in (mechanical and electrical) contact with the bottom metal layer of the rectifier. The selected antennas in this frequency band are mainly based on dipole [3]-[5] or annular slot topologies [7]. The patch antennas are impractical in ISM 868/915 MHz band due to the large size. Flat dipole antennas topologies were selected in the past mainly for their broadband and/or multiband behavior [3]-[4]. We selected this topology here because of (i) its broadband behavior that allows operating efficiently in the ISM $868 \mathrm{MHz}$ (Europe) and 915 $\mathrm{MHz}$ (USA/Japan) bands and, (ii) the large metal surface of the radiating element that allows integrating the rectifier and eventually other electronic devices. The flat dipole can be miniaturized (the size for operating at the targeted frequency is reduced compared with one of the standard flat dipoles) by adding an external ring/loop properly designed (see Fig.2). In our case, the size was reduced by $25 \%$ (from to $14 \times 6 \mathrm{~cm}^{2}$ to $10.5 \times 6 \mathrm{~cm}^{2}$ ). The topology of the rectifier (with the dimensions of microstrip lines) and corresponding ADS simulation model are shown in Fig. 3.

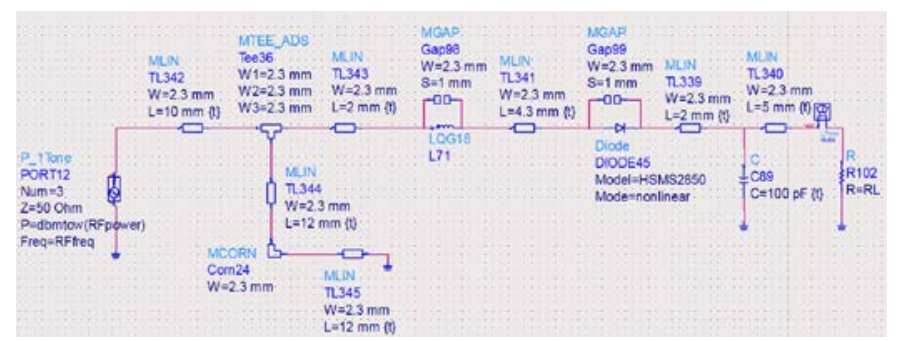

Fig. 3. Rectifier topology and associated ADS simulation model

The rectifier is composed of a series HSMS2850 diode from Avago, a shunt capacitor $(100 \mathrm{pF})$ and a resistive load $(10 \mathrm{k} \Omega)$. This rectifier was previously designed, fabricated and characterized in our Laboratory in the framework of a research project with UWINLOC company [2]. An impedance matching circuit combining a short-circuited bend stub with a $30 \mathrm{nH}$ inductance (Coilcraft) was used in order to match the impedance of the rectifier at $900 \mathrm{MHz}$. The rectifier was fabricated on Ro5870 substrate (thickness: $0.787 \mathrm{~mm}$, relative permittivity: 2.3, dielectric loss tangent: 0.0012) and optimized to operate efficiently for low electromagnetic input power (less than $-15 \mathrm{dBm}$ ).

\section{EXPERIMENTAL AND SIMULATION RESULTS}

The experimental setup positioned in an anechoic chamber (to prevent any interferences or undesirable multipath effects) shown in Fig. 4 was used to characterize the rectenna. A microwave signal generated from the Anritsu MG3694B generator is injected at the input of a transmitting (Tx) horn antenna through a coaxial cable. The horn antenna illuminates the rectenna under test, positioned in the far-field region of the Tx antenna, with a linearly-polarized E-field. An automatic acquisition routine is implemented in Labview software from National Instruments to speed-up the acquisition process. The harvested DC voltage is then measured by using a DC multimeter. The DC power can be derived from the measured DC voltage as long as the load is known. The efficiency $\eta$ (in \%) of the rectenna can be computed by using the following expression:

$$
\eta=\frac{P_{D C}}{P_{R F}} \cdot 100=\frac{P_{D C}}{S \cdot A_{e f f}} \cdot 100=\frac{4 \cdot \pi \cdot P_{D C}}{S \cdot G_{R} \cdot \lambda^{2}} \cdot 100
$$

where $P_{D C}$ is the harvested DC power, $S$ is the incident electromagnetic power density, $A_{e f f}$ is the antenna effective area, $G_{R}$ is the gain of the (rectenna's) antenna and $\lambda$ is the free-space wavelength of the illuminating electromagnetic wave at the operating frequency. The electromagnetic power density $S$ can be computed as a function of the E-field 
effective value $E(\mathrm{~V} / \mathrm{m})$ on the antenna surface. This value is derived from microwave power $P_{t}$ injected to the input port of the transmitting horn antenna (gain $G_{t}$ ) positioned at a distance $d$ from the rectenna, as follows:

$$
S=\frac{E^{2}}{120 \cdot \pi}=\frac{30 \cdot P_{t} \cdot G_{t}}{d^{2} \cdot 120 \cdot \pi}
$$

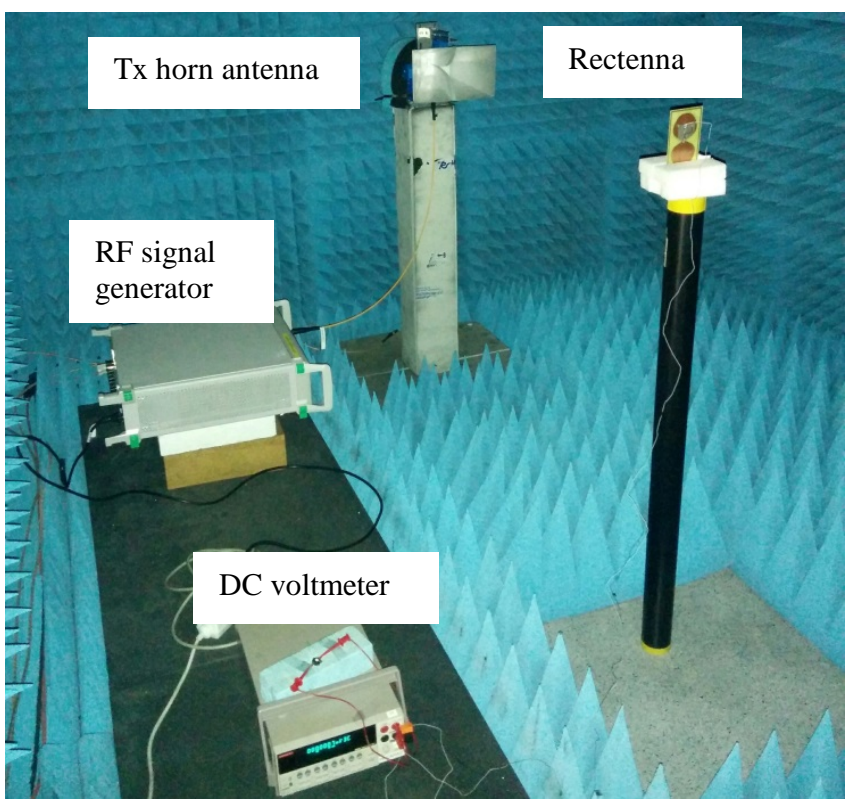

Fig. 4. Experimental setup used to characterize the manufactured rectenna.

The flat round antenna was fabricated on a low-cost FR4 substrate (substrate thickness: $0.8 \mathrm{~mm}$, relative permittivity: 4.4, dielectric loss tangent: 0.02). As shown in Fig. 5(a), the simulated (HFSS) $-10 \mathrm{~dB}$ bandwidth of the antenna is between $845 \mathrm{MHz}$ and $1.25 \mathrm{GHz}$ and consequently, covers the ISM 868/915 MHz bands. For comparison purposes the reflection coefficient at the antenna input with and without the rectangular ring are depicted in Fig. 5(a).

We can observe that the ring increases the bandwidth and allows reducing the size of the antenna. Electromagnetic simulations were performed in order to optimize the rectangular ring. The ring perimeter $(33 \mathrm{~cm})$ and the distance between the ring and flat dipole antenna were adjusted in order to: (i) minimize the lowest operating frequency and (ii) increase the frequency bandwidth. The radiation pattern is close to one of the half-wavelength dipole with a simulated gain of $2.2 \mathrm{dBi}$ in the $-10 \mathrm{~dB}$ bandwidth (845 MHz-1.25 GHz). A prototype composed by a flat dipole antenna (enclosed by a rectangular metallic ring) and a compact printed taper were measured. The experimental results reported in Fig. 5(b) validate the frequency bandwidth predicted by the electromagnetic simulations.

The fabricated rectenna (see Fig. 2 d) was characterized by using the experimental setup shown in Fig. 4. The measured DC output voltage delivered by the manufactured rectenna on a non-optimal load of $10 \mathrm{k} \Omega$ is reported in Fig. 6. The maximum DC voltage $(471 \mathrm{mV})$ is obtained at $860 \mathrm{MHz}$ for an incident E-field $E=1.28 \mathrm{~V} / \mathrm{m}\left(0.44 \mu \mathrm{W} / \mathrm{cm}^{2}\right)$. At $868 \mathrm{MHz}$, the DC voltage is about $450 \mathrm{mV}\left(0.44 \mu \mathrm{W} / \mathrm{cm}^{2}\right)$.
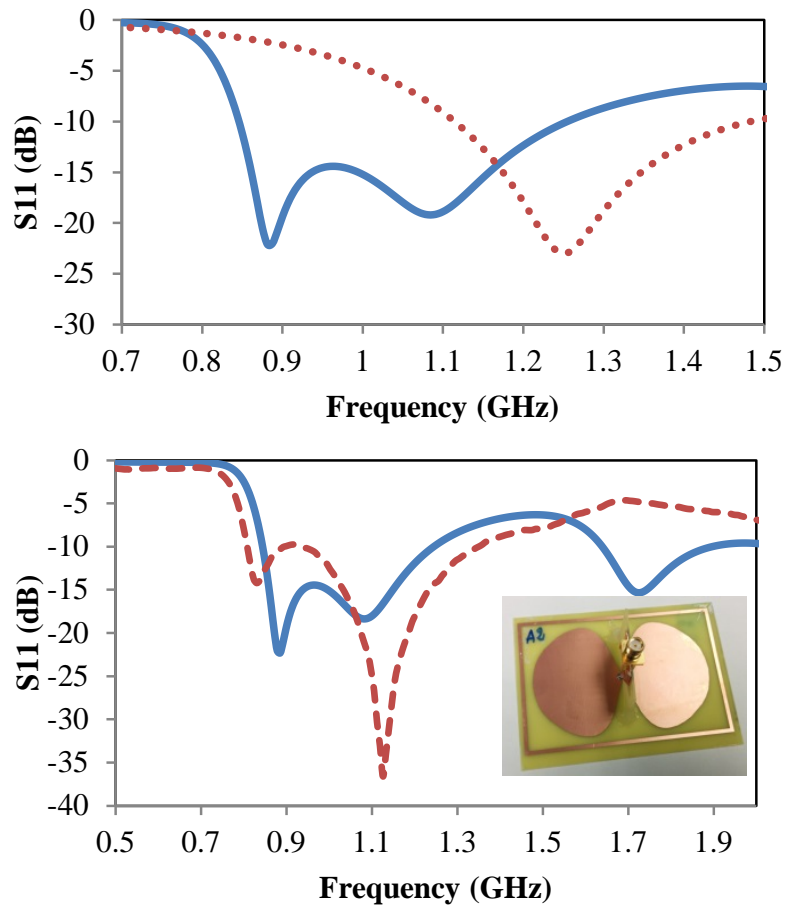

Fig. 5. (a) Simulated (HFSS) reflection coefficient (S11 in dB) as a function of the frequency for the flat dipole antenna enclosed by the rectangular ring (continuous blue line) and without ring (dotted red line); (b) Simulated reflection coefficient of the flat dipole antenna enclosed by the rectangular ring (continuous blue line) and the measured reflection coefficient of the fabricated prototype (dotted red line). The inset shows a photo of the manufactured antenna including the compact taper.

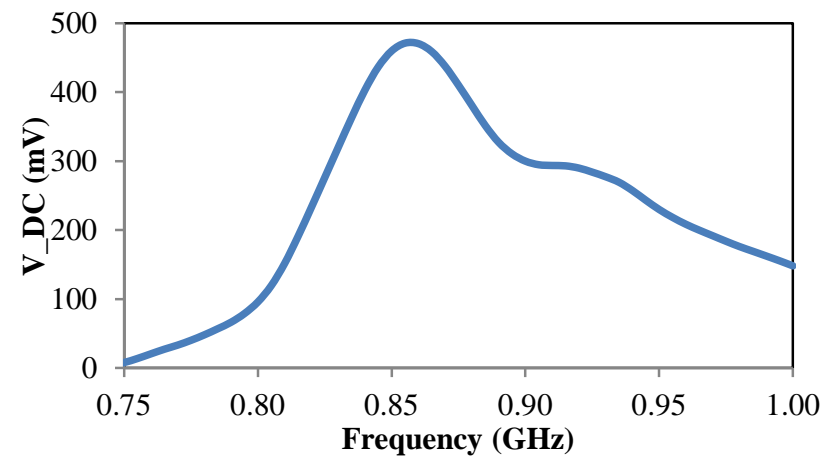

Fig. 6. Measured harvested DC voltage (V_DC) for the manufactured rectenna (incident E-field: $1.28 \mathrm{~V} / \mathrm{m}$, load: $10 \mathrm{k} \Omega$ ) as a function of frequency.

The measured DC voltage and efficiency computed by using eq. (1) are given in Fig. 7 as a function of the incident E-field amplitude. A maximum efficiency of 37\% (DC voltage: 1.13 V and DC power: $130 \mu \mathrm{W})$ is obtained for $\mathrm{E}=2.88 \mathrm{~V} / \mathrm{m}(2.2$ $\mu \mathrm{W} / \mathrm{cm}^{2}$ ). As compared with the 3D rectenna [9] shown in Fig. 2(a) the 2D rectenna exhibits a little bit lower efficiency, but it is more compact, planar and compatible with a multilayer fabrication process. 


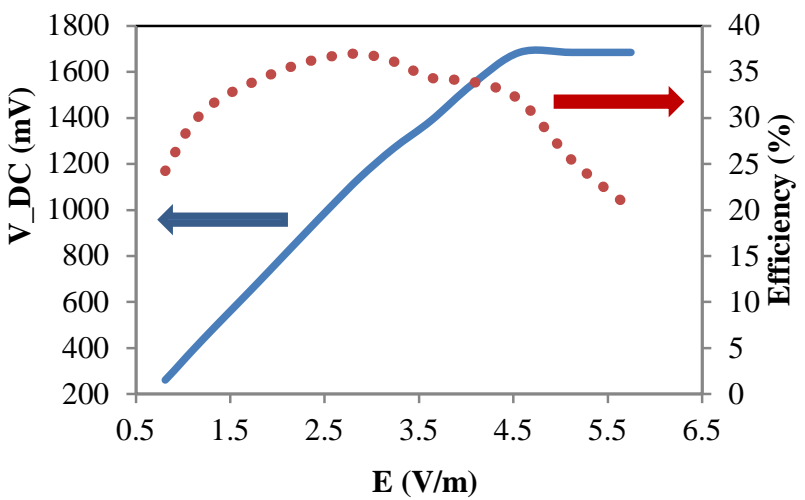

Fig. 7. Measured harvested DC voltage (V_DC: continuous blue line, values on vertical left axis) and efficiency (dotted red line, values in \% on vertical right axis) as a function of incident E-field (frequency $=860 \mathrm{MHz}$, load: $10 \mathrm{k} \Omega$ )

A comparison with the recent state-of-art rectennas operating in the same frequency band is reported in Table 1. We selected only designs that use planar antennas and papers that reports experimental results for low-level of incident microwave power density. The state-of-art performances are reported as a function of the estimated microwave power on the rectifier input PRF or as a function of the incident microwave power density $S$. The size of the antennas/rectennas are reported in $\mathrm{cm}^{2}$ or as function of square wavelength $\left(\lambda^{2}\right)$ allowing thus a fair comparison between designs operating at different frequencies. As reported in Table 1 the state-of-art designs use: (i) 3D topologies (the rectifier is interconnected with the antenna in a 3D manner as reported in [3]-[4]), (ii) planar but inline (IL) design that typically conducts to a 'large-size' design (e.g. [5]) (iii) planar designs where the rectifier is integrated in the radiating element (this work) or connected by wire and positioned on the back-side of the antenna [7] that lead to a more compact designs.

Table 1. Comparison with state-of-the-art designs.

\begin{tabular}{|c|c|c|c|c|c|}
\hline Ref & $\begin{array}{c}\text { Freq } \\
\text { (GHz) }\end{array}$ & $\begin{array}{l}\mathrm{S}\left(\mu \mathrm{NW} / \mathrm{cm}^{2}\right) \\
\mathrm{PRF}(\mathrm{dBm})\end{array}$ & $\eta(\%)$ & $\begin{array}{c}\text { Antenna } \\
\text { Surface }\end{array}$ & $\begin{array}{l}\text { Rectenna } \\
\text { Surface }\end{array}$ \\
\hline [3] & 0.9 & $-15 \mathrm{dBm}$ & 30 & NR & NR/3D* \\
\hline$[5]$ & 0.868 & $\begin{array}{c}0.1 \mu \mathrm{W} / \mathrm{cm}^{2} \\
1 \mu \mathrm{W} / \mathrm{cm}^{2}\end{array}$ & $\begin{array}{c}30 \\
44.8\end{array}$ & NR & NR/IL** \\
\hline [10] & 0.9 & $-10 \mathrm{dBm}$ & 33 & NR & NR \\
\hline [7] & 0.9 & $\begin{array}{c}0.1 \mu \mathrm{W} / \mathrm{cm}^{2} \\
1 \mu \mathrm{W} / \mathrm{cm}^{2}\end{array}$ & $\begin{array}{l}16 \\
40\end{array}$ & $\begin{array}{c}11 \times 11 \mathrm{~cm}^{2} \\
0.11 \lambda^{2}\end{array}$ & $\begin{array}{l}11 \times 11 \mathbf{c m}^{2} \\
0.11 \lambda^{2} * * *\end{array}$ \\
\hline $\begin{array}{l}\text { This } \\
\text { work }\end{array}$ & 0.86 & $\begin{array}{c}0.21 \mu \mathrm{W} / \mathrm{cm}^{2} \\
1.1 \mu \mathrm{W} / \mathrm{cm}^{2} \\
2.2 \mu \mathrm{W} / \mathrm{cm}^{2}\end{array}$ & $\begin{array}{c}31 \\
35.5 \\
37 \\
\end{array}$ & $\begin{array}{c}10.5 \times 6 \mathrm{~cm}^{2} \\
0.05 \lambda^{2}\end{array}$ & $\begin{array}{c}10.5 \times 6 \mathrm{~cm}^{2} \\
0.05 \lambda^{2}\end{array}$ \\
\hline
\end{tabular}

NR : not reported in the pape

* : rectifier connected to the antenna in an orthogonal/3D manne

** : rectifier connected to the antenna by an In Line (planar) connection

*** a wire-connected multiband-rectifier is positioned on the back-side of the antenna

It can be observed from Table 1 that the energy harvesting performances of the proposed rectenna are in the state-of-theart, but with a more compact structure: the antenna/rectenna surface is of $10.5 \times 6 \mathrm{~cm}^{2}$, that is, only $0.05 \lambda^{2}$ at $860 \mathrm{MHz}$. We note also that the efficiencies were measured for optimal loads while in this work, the load is non-optimal. As a matter of fact, the selected (non optimal) load of $10 \mathrm{k} \Omega$ allows: (i) to maximize the DC output voltage required for the cold startup/wake-up of a commercial off-the-shelf DC-to-DC boost converter and, (ii) to simulate the input impedance of the same DC-to-DC boost converter. There is room on our antenna surface to integrate more electronic devices (the metal layer of flat dipole antenna is quite large) in order to obtain a miniaturized autonomous wireless sensor/tag. Moreover, depending on the targeted market, the overall structure can be manufactured either on a multilayer low cost (e.g., FR4) or on low loss (e.g., Ro5870) substrate.

\section{CONCLUSION}

An innovative compact rectenna $\left(0.05 \lambda^{2}\right.$ at $\left.860 \mathrm{MHz}\right)$ was proposed. It consists of a miniaturized flat dipole antenna with a rectifier which was optimized to operate at low microwave power densities. The rectifier is directly integrated into the surface of the radiating element. The large size of the metal layer of the flat dipole is very convenient for the eventual integration of additional electronic devices. A maximum efficiency of $37 \%$ is measured at $860 \mathrm{MHz}$ for the manufactured rectenna with an illuminated electromagnetic power density of $2.2 \mu \mathrm{W} / \mathrm{cm}^{2}$ only.

\section{ACKNOWLEDGMENT}

LAAS CNRS wishes to acknowledge UWINLOC company and the support of the French region OCCITANIE through the research project OPTENLOC.

\section{REFERENCES}

[1] https://www.enocean.com/en/technology/self-powered-internet-ofthings/

[2] http://uwinloc.com/\#innovation

[3] V. Kuhn, C. Lahuec, F. Seguin, and C. Person, “A Multi-Band Stacked RF Energy Harvester With RF-to-DC Efficiency Up to 84\%,” IEEE Trans. Microw. Theory Tech., vol. 63, no. 5, pp. 1768-1778, May 2015

[4] C. Song, Y. Huang, J. Zhou, J. Zhang, S. Yuan, and P. Carter, “A high efficiency broadband rectenna for ambient wireless energy harvesting,’IEEE Trans. Antennas Propag., vol. 63, no. 8, pp. 34863495, Aug. 2015.

[5] S. D. Assimonis, S. N. Daskalakis, and A. Bletsas, "Sensitive and Efficient RF Harvesting Supply for Batteryless Backscatter Sensor Networks,” IEEE Trans. Microw. Theory Tech., vol. 64, no. 4, pp. 13271338, Apr. 2016.

[6] Z. Popovic; E.A. Falkenstein, D. Costinett, R. Zane, "Low-Power FarField Wireless Powering for Wireless Sensors", Proceedings of the IEEE, Vol. 101, No. 6, pp.1397 -1407, June 2013.

[7] V. Palazzi, J. Hester, J. Bito, F. Alimenti, , C. Kalialakis, A. Collado, P. Mezzanotte, A. Georgiadis, L. Roselli, M. M. Tentzeris, 'Novel UltraLightweight Multiband Rectenna on Paper for RF Energy Harvesting in the Next Generation LTE Bands', IEEE Trans. Microw. Theory Tech., vol. pp, issue 99 (early access paper)

[8] http://www.ti.com/product/BQ25504

[9] A. Takacs, A. Okba, H. Aubert, “900 MHz Miniaturized Rectenna”, (under soumission)

[10] D. Masotti, A. Costanzo, P. Francia, M. Filippi, and A. Romani, “A Load-Modulated Rectifier for RF Micropower Harvesting With StartUp Strategies,” IEEE Trans. Microw. Theory Tech., vol. 62, no. 4, pp. 994-1004, Apr. 2014. 